\title{
One-Dimensional Discontinuous Flows in Relativistic Magnetohydrodynamics
}

\author{
V.I. ZHDANOV and P.V. TITARENKO \\ Astronomical Observatory of Kyiv Schevchenko University, \\ 3 Observatorna Street, 254053 Kyïv, Ukraïna, \\ E-mail: zhdan@aoku.gluk.apc.org
}

\begin{abstract}
We consider discontinuous flows of relativistic magnetic fluid with a general equation of state that is not supposed to be normal in the sense of Bethe and Weyl. The criteria of admissibility of the shock waves without a supposition of the relativistic version of the convexity condition are obtained. The results are used to analyze the cases of perpendicular and parallel shock waves.
\end{abstract}

1. It is well known that the uniqueness of solutions of an initial value problem for quasilinear equations such as equations of fluid dynamics may be lost if discontinuities of initial data are present [1]. In this case some additional requirements are needed to single out a unique discontinuous solution. In hydrodynamics with a normal equation of state (in the sense of Bethe and Weyl [1]) the role of such a requirement is fulfilled by the entropy criterion. In case of anomalous fluid with a nonconvex equation of state some more stringent criteria are needed that may be represented as requirements to the form of shock adiabate (see, e.g., [2], [3]). The generalization to the relativistic shock waves is worked out in [4], [5].

In this paper the analogous problem in relativistic magnetohydrodynamics is considered. In order to formulate the conditions for existence of admissible shock waves in the fluid with a nonconvex (in the relativistic sense) equation of state we modify the approach of [4], [5] based on the small viscosity considerations. The resulting criterion is expressed in terms of shock adiabats, defined by the equation of state, and some pattern curves in the plane of thermodynamic coordinates pressure-conserving charge density. This criterion is applied to study parallel and perpendicular shocks in a one-dimensional magnetohydrodynamic flow.

We start from the equations of motion of a viscous relativistic fluid with perfect electrical conductivity, permeated by a magnetic field [6], [7]

$$
\begin{aligned}
& \partial_{\mu}\left(T^{\mu \nu}+\tau^{\mu \nu}\right)=0, \\
& \partial_{\mu}\left(u^{\mu} h^{\nu}-u^{\nu} h^{\mu}\right)=0, \\
& \partial_{\mu}\left(n u^{\mu}\right)=0, \\
& h^{\mu} u_{\mu}=0, \\
& \tau^{\mu \nu} u_{\nu}=0 ;
\end{aligned}
$$


where

$$
T^{\mu \nu}=\left(p^{*}+\varepsilon^{*}\right) u^{\mu} u^{\nu}-p^{*} g^{\mu \nu}-\frac{1}{4 \pi} h^{\mu} h^{\nu}
$$

is the magnetohydrodynamic relativistic energy-momentum tensor with $u^{\mu}$ being 4 -velocity, $h^{\mu}=-\frac{1}{2} e^{\mu \alpha \beta \gamma} F_{\alpha \beta} u_{\gamma}$ being 4 -vector of the magnetic field ( $F_{\alpha \beta}$ is the tensor of electromagnetic field here) and $p^{*}=p+\frac{1}{8 \pi}|h|^{2}, \varepsilon^{*}=\varepsilon+\frac{1}{8 \pi}|h|^{2}, p$ is the pressure and $\varepsilon$ is the specific energy density, $n$ is the density of some conserved charge, $\partial_{\mu}=\partial / \partial x^{\mu}$ and

$$
\tau_{\mu \nu}=\eta\left(u_{\mu, \nu}+u_{\nu, \mu}-u_{\mu} u^{\alpha} u_{\nu, \alpha}-u_{\nu} u^{\alpha} u_{\mu, \alpha}\right)+\left(\zeta-\frac{2}{3} \eta\right) \frac{\partial u^{\alpha}}{\partial x^{\alpha}}\left(g_{\mu \alpha}-u_{\mu} u_{\nu}\right)
$$

is the relativistic viscosity tensor [6].

We assume that the equation of state $p=p(V, \varepsilon)$ is represented by a sufficiently smooth function.

In the limit of small viscosity we expect that solutions of hydrodynamic equations yield the discontinuous flows describing shock waves.

It is sufficient to confine our consideration to one-dimensional fluid motion along the $x$ axis. In the rest frame of the discontinuity located at the surface $x=0$ the following conditions hold

$$
\left\{n u^{1}\right\}=0, \quad\left\{T^{1 \beta}\right\}=0
$$

(conservation of current and energy-momentum),

$$
\left\{u^{1} h^{\beta}-u^{\beta} h^{1}\right\}=0
$$

(continuity of the normal component of magnetic field and tangential component of electric field).

Here $\{A\}=A_{1}-A_{0}$, where $A_{1}$ is a quantity ahead of the shock and $A_{0}$ is a quantity behind the shock. It is easy to see that $u_{(0),(1)}^{3}$ and $h_{(0),(1)}^{3}$ may be taken zero.

From conservation laws and the Maxwell equations one obtains the equations that relate the sets of hydrodynamic and electrodynamic quantities $u_{(0)}^{\mu}, n_{0}, p_{0}$ and $u_{(1)}^{\mu}, n_{1}, p_{1}$ on both sides of the shock. Both sets are located on the magnetohydrodynamic analogue of the Taub adiabat defined by the equation [7]:

$$
\mathbf{H} \equiv w^{2} V^{2}-w_{0}^{2} V_{0}^{2}+\left(w V^{2}+w_{0} V_{0}^{2}\right)\left(p-p_{0}\right)+\frac{1}{2}\left(w V^{2}-w_{0} V_{0}^{2}\right)\left(\psi-\psi_{0}\right)^{2}=0,(8)
$$

where $w=\varepsilon+p, \psi^{2}=h_{n}^{2}+|h|^{2}\left(1-u_{n}^{2}\right)$. We assume that:

(a) the equation (8) has unique solution with respect to $p$ in all domain of parameters changing;

(b) the Bethe assumption $(\partial p / \partial s)_{n}>0$ holds.

We shall investigate the boundary problem that describes a shock wave in the limit of viscosity tending to zero. The existence conditions of the solution of this problem are then interpreted as the conditions of admissibility of the shock transition $u_{(0)}^{\mu}, n_{0}, p_{0} \rightarrow$ $u_{(1)}^{\mu}, n_{1}, p_{1}$, where the corresponding quantities satisfy the relations (6)-(7) on the shock wave. 
2. Consider stationary motion of fluid with all the quantities depending only on $x$ and $u^{3}=0, h^{3}=0$. The equations (1)-(3) give the following

$$
\begin{aligned}
& T^{1 \nu}+\tau^{1 \nu}=\text { const, } \\
& u^{1} h^{\nu}-h^{1} u^{\nu} \equiv H^{\nu}=\mathrm{const}, \\
& n u^{1} \equiv j=\text { const. }
\end{aligned}
$$

For the set (9)-(11), (4)-(5) we assume that under $x \rightarrow \pm \infty$ all quantities tend to some constant values, which are marked by " 0 " and " 1 ". It is easy to see that points "0" and "1" lay on shock adiabat (8). If the viscosity tends to zero, we obtain the conditions (6)-(7) exactly.

In order to simplify the problem we take $\eta$ to be zero. Equations (9) yield

$$
\begin{aligned}
& \zeta\left(1+\left(u^{1}\right)^{2}\right) \frac{d u^{1}}{d x}=T^{11}-T_{(0)}^{11}, \\
& \varepsilon^{*} u^{1}=T_{(0)}^{1 \mu} u_{\mu}, \\
& \left(H^{2} u^{2}-H^{0} u^{0}\right)\left(H^{2} u^{0}-H^{0} u^{2}\right)=4 \pi u^{1}\left(T_{(0)}^{10} u^{2}-T_{(0)}^{12} u^{0}\right),
\end{aligned}
$$

where $H^{2} u^{2}-H^{0} u^{0} \equiv h^{1}$.

The last equation can be easily studied after rewriting it in terms of the threedimensional velocity components $v_{1}=u^{1} / u^{0}, v_{2}=u^{2} / u^{0}$. The result of the investigation shows that there are two physical branches of the curve $v_{2}\left(v_{1}\right)$ corresponding to "fast" and "slow" shock waves. Further we suppose that we are working on a connected component of this curve.

The set of equations (10)-(13), and (4) gives our solution to be found. And we have to find under which conditions this solution exists. Note that only differential equation (14) may be written as

$$
\zeta \frac{d V}{d x}=p(V, \widetilde{\varepsilon}(V))-\widetilde{p}(V),
$$

where the function $\widetilde{p}(V)$ is determined through the equations (10), (11), (13) and $\widetilde{\varepsilon}(V)$ by (12). From the necessary and sufficient conditions of existence of solution of equation (14) with boundary conditions $u_{(1)}^{\mu}, p_{1}, n_{1}$ for $x \rightarrow+\infty$ and $u_{(0)}^{\mu}, p_{0}, n_{0}$ for $x \rightarrow-\infty$, we obtain the following criterion of admissibility of shock transition:

(c) the equation (13) has a regular solution $u^{2}=u^{2}(V)$ between $V_{0}$ and $V_{1}$;

$$
\text { (d) } \quad\left(V_{1}-V_{0}\right)(p(V, \widetilde{\varepsilon}(V))-\widetilde{p}(V)) \geq 0
$$

for all $V$ between $V_{0}$ and $V_{1}$.

The following theorem states the other form of criterion where $p(V, \widetilde{\varepsilon}(V))$ is changed by shock adiabat.

Theorem. Let the assumptions (a), (b) hold. Then the condition (15) is equivalent to the condition

$$
\left(V_{1}-V_{0}\right)\left(p_{H}(V)-\widetilde{p}(V)\right) \geq 0
$$


with the shock adiabat $p_{H}(V)$ determined from the equation (8).

3. The pattern function $\widetilde{p}(V)$ can be easily derived for the special cases of parallel and perpendicular shock waves. For the parallel shock wave $u^{2}=0, h^{2}=0$ and for perpendicular that $u^{2}=0, h^{1}=0$. Consequently, $H^{2}=0$ and $H^{0}=0$, respectively. For both cases equation (13) is satisfied identically.

For the parallel shock wave we obtain the pattern function

$$
\widetilde{p}(V)=\frac{1}{8 \pi}\left(\frac{h_{(0)}^{1}}{u_{(0)}^{0}}\right)^{2}+T_{(0)}^{11}-\frac{T_{(0)}^{10} j V}{\sqrt{1+j^{2} V^{2}}} .
$$

Note that the condition $(c)$ for the solution of (14) in case of the parallel shock wave also must be taken into account though (13) is satisfied trivially by $u^{2}=0$. This restricts the domain of possible shock transitions.

For the perpendicular shock wave we obtain

$$
\widetilde{p}(V)=T_{(0)}^{11}-\frac{T_{(0)}^{10} j V}{\sqrt{1+j^{2} V^{2}}}-\frac{1}{4 \pi} \frac{\left(u_{(0)}^{1} h_{(0)}^{2}\right)^{2}}{j^{2} V^{2}} .
$$

In this case the condition $(c)$ leads to no restrictions. The solution of an arbitrary decay problem can be obtained here in analogy to [8], [9].

\section{References}

[1] Rozhdestvensky B.L., Yanenko N.N., Systems of quasilinear equations, Moscow, Nauka, 1978, 687p. (in Russian).

[2] Wendroff B., The Riemann problem for materials with nonconvex equation of state, J. Math. Anal. Appl., 1972, V.38, N 2, 454-466; N 3, 640-658.

[3] Sidorenko A.D., Wave adiabats for the medium with arbitrary equation of state, DAN SSSR, 1968, V.178, N 4, 818-821 (in Russian).

[4] Bugaev K.A., Gorenstein M.I., Zhdanov V.I., Relativistic shocks in the systems containing domains with anomalous equation of state and quark baryonic matter hadronization, Z. Phys. C, 1988, V.39, $365-370$.

[5] Bugaev K.A., Gorenstein M.I., Zhdanov V.I., Relativistic shock waves in presence of domains with anomalous equation of state, Zh. Theor. Math. Phys.,, 1989, V.80, N 1 (in Russian).

[6] Landau L.D., Lifshitz E.M., Hydrodynamics, Moscow, Nauka, 1986, 733p. (in Russian).

[7] Lichnerovicz A., Relativity theory and mathematical physics, In: Astrophysics, Quantum and Relativity Theory, Mir, Moscow, 1974 (in Russian).

[8] Bugaev K.A., Gorenstein M.I., Kämpfer B., Zhdanov V.I., Generalized shock adiabatics and relativistic nuclear collisions, Phys. Rev. D, 1989, V.40, 2903.

[9] Zhdanov V.I., Kolomoets S.V., One-dimensional discontinuous flows of the relativistic fluid with anomalous equation of state, Visnyk Kyivs'kogo Universitetu (Astronomy), 1994, N 33, 31-34. 